\title{
A Fast and Accurate Iris Segmentation Approach
}

\author{
Guojun Cheng ${ }^{1}$, Wenming Yang ${ }^{1}$, Dongping Zhang ${ }^{2}$, and Qingmin $\operatorname{Liao}^{1(\bowtie)}$ \\ 1 Shenzhen Key Laboratory of Information Science and Technology/Shenzhen \\ Engineering Laboratory of Information Security, DRM Department of Electronic \\ Engineering/Graduate School at Shenzhen, Tsinghua University, Beijing, China \\ liaoqm@tsinghua.edu.cn \\ ${ }^{2}$ College of Information Engineering, China Jiliang University, Hangzhou, China \\ 06A0303103@cjlu.edu.cn
}

\begin{abstract}
Iris segmentation is a vital forepart module in iris recognition because it isolates the valid image region used for subsequent processing such as feature extraction. Traditional iris segmentation methods often involve an exhaustive search in a certain large parameter space, which is time consuming and sensitive to noise. Compared to traditional methods, this paper presents a novel algorithm for accurate and fast iris segmentation. A gray histogram-based adaptive threshold is used to generate a binary image, followed by connected component analysis, and rough pupil is separated. Then a strategy of RANSAC (Random sample consensus) is adopted to refine the pupil boundary. We present Valley Location of Radius-Gray Distribution (VLRGD) to detect the weak iris outer boundary and fit the edge. Experimental results on the popular iris database CASIA-Iris V4-Lamp demonstrate that the proposed approach is accurate and efficient.
\end{abstract}

Keywords: Biometrics $\cdot$ Iris segmentation $\cdot$ Adaptive threshold $\cdot$ Iris boundary detection $\cdot$ Edge fitting

\section{Introduction}

With the increasing demands of security in our everyday life, biometric technology plays a more and more significant and irreplaceable role. It mainly utilizes the physical and behavior characteristics, which include face, iris fingerprint, finger vein, voice, handwriting, and so on, to verify and identify individuals. Among all the biometric characteristics, iris is one of the most unique patterns for advantages that it displays rich and detailed textures of high degrees of freedom, and remains stable over a lifetime $[1,2]$.

A typical iris recognition systems comprises the following fundamental modules: eye image acquisition (shown as Fig. 1), iris localization and segmentation, normalization, feature extraction, and template matching [3]. In the segmentation stage, the iris is segmented from the eye image captured by cameras under visible or near-infrared illumination, which aims at extracting the valid part of 
the iris and isolating it from other tissues, such as pupil, sclera, eyelid, eyelashes or reflections. So iris segmentation generally includes pupillary boundary and iris outer boundary detection, eyelids and eyelashes exclusion and specular removal. This step plays a key role in the entire system and is even considered the most significant part because it strongly affects the accuracy of the recognition rate.

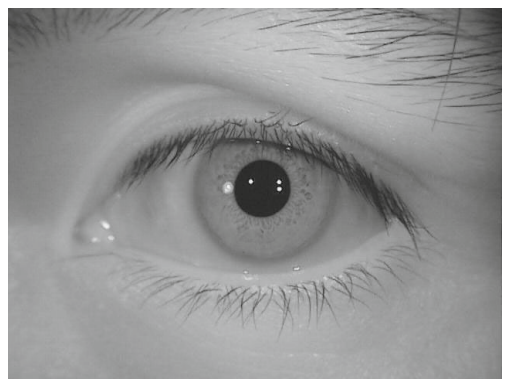

Fig. 1. Iris image

\subsection{Related Work}

Many researchers proposed many powerful iris recognition algorithms, which can be classified into two categories [3]: circle/ellipse model such as the well-known integro-differential operator by Daugman [2,7], Hough Circle Transform after edge detection by Wildes [11], or their derivative [4-10,12-16] and non-ideal model, like Active Contour model followed by Fourier series expansion [17], and Pulling and Pushing iteratively followed by polynomial fitting [18]. The first two algorithms work efficiently on ideal and high-quality image with good-contrast, but there are an exhaustive search in a certain large parameter space to get the parameters of inner and outer of iris, which is time-consuming and sensitive to occlusion by reflections, eyelids or eyelashes and most importantly fail in noncircular likeness iris boundary such as off-angle iris. The Hough Circle Transform model exploiting gradient amplitude information to vote, is sensitive to local extrema caused by reflections, especially at boundaries. The AC model with the curve evolution time consuming, is badly sensitive to noises too, especially reflections and disturbances among boundaries and may lead to oversegmentation under weak iris edges. In the PP method, it's really hard to select the three stable points on the weak and noisy iris edge.

It's said in previous work that a fast and accurate pupil localization method will contribute much to iris segmentation procedure [14,19]. Generally speaking, a pupil localization method includes eye image de-noising, thresholding, pupil boundary detection, and pupil center localization. But the threshold for pupil binary is always selected as a fixed value, which is not reasonable or efficient.

Based on the review above, we present a new method to select a relatively proper threshold adaptively according to the gray-level histogram after specular 
removal, which leads to good results with connected component analysis and refined phase by RANSAC [9]. It's self-evident that our approach can be adapted to the noncircular case. As to the weak iris outlier, a method called Valley Location of Radius-Gray Distribution (VLRGD) is adopted and the edge is then fitted. After that, a local minimum filter is used to decrease eyelash noises and then eyelids are localized.

The rest of this paper is organised as follows: in Sect. 2, proposed algorithms are described detailed and in Sect.3, the experiment results and performance of proposed methods with the CASIA-Iris V4-Lamp iris database [23] are illustrated. In Sect. 4, we make the conclusions.

\section{The Principle of Proposed Method}

In the following subsections, we divide the whole algorithm into three stages: reflection removal, pupil detection including adaptive thresholding, connected component analysis and pupil refinement, and iris outlier detection including edge points getting and boundary fitting. The framework of the proposed algorithm is shown in Fig. 2.

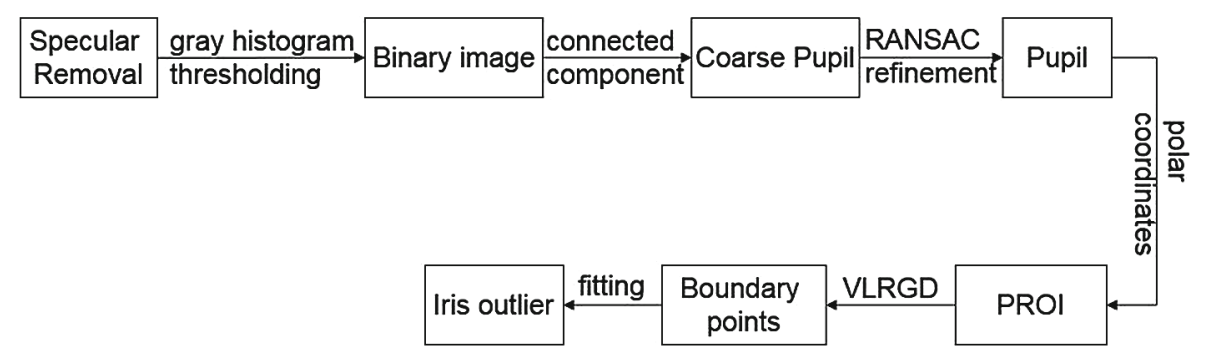

Fig. 2. Framework of proposed algorithm

\subsection{Reflection Removal}

Specular reflections usually appear as the brightest pixels in the iris image $I(x, y)$ [18]. As the bright reflections are always located in the dark pupil or in the less darker iris region, there is always a higher contrast in the local regions around them. Based on this observation, we consider the brightest $\mathrm{p} 1$ pixels with relative higher local gray-level contrast as reflections, where p1 is quiet flexible to choose and can be even set to 0.6 in this work to ensure all the reflections are included (shown in Fig. 3(a)).

Then in the candidate 'reflections', we check the gray-level contrast determinated by Eq. (1):

$$
G C=\frac{I(x, y)}{\frac{1}{(2 p+1)^{2}-1} \sum_{j \in \Omega} \sum_{i \in \Omega} I(x+i, y+j)}, \Omega=\{-p, \ldots,-1,1, \ldots, p\} .
$$




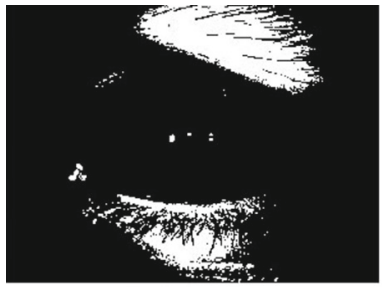

(a)

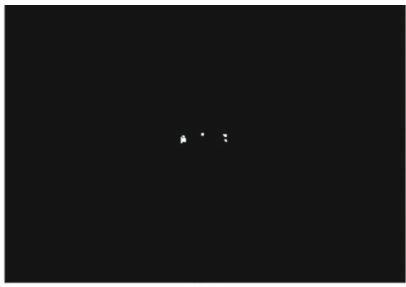

(b)

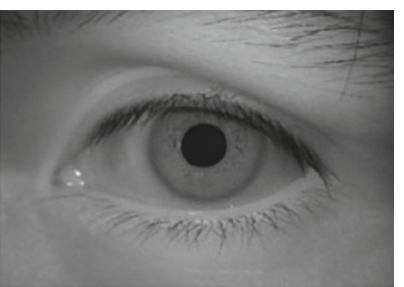

(c)

Fig. 3. Specular removal. (a) Binary, p1 $=60 \%$, (b) Detected specular, (c) Denoised eye image.

GC is the ratio of the central gray-level to the average value of other neighbour pixels. The p should be big enough to ensure the neighbour of reflections cover the specular reflections region (shown in Fig. 3(b)). We call the denoised eye image $I_{a}(x, y)$ (shown in Fig. 3(c)).

\subsection{Pupillary Boundary Detection}

\subsubsection{Gray Histogram-Based Adaptive Threshold}

As illustrated above, pupil appears as the darkest region in the eye image as well as some eyelashes and hairs. The gray-level histogram of the eye image is shown in Fig. 4, in which the first peak in the left represents the darkest pixels including the pupil ((b) is the part of dotted box in (a)).

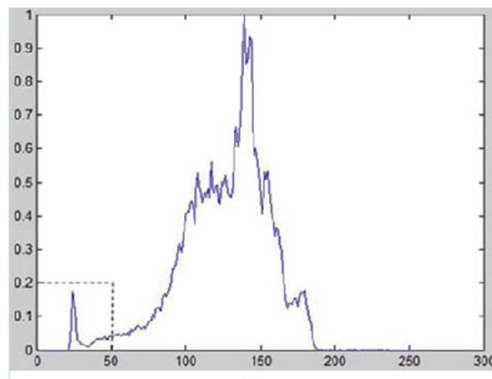

(a)

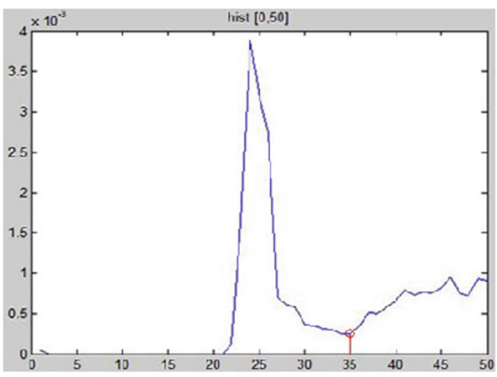

(b)

Fig. 4. Gray-level histogram of the eye image. (a) the whole histogram. (b) the local part in the dotted box of (a), the horizontal ordinate of the red vertical line is the adaptive threshold $\mathrm{T}$.

We choose the gray value of the valley between the first two peaks as the threshold $\mathrm{T}$ adaptively to binary the iris image. To calculate the $\mathrm{T}$, we get the first peak as Hist $[p]$ in the histogram of $[0,50]$ and the local minimum values as $\{\text { Hist }[b[i]]\}_{i=1}^{\text {Num }}$ in the histogram of $(\mathrm{p}, 50]$, then calculate the absolute slope of the peak and each of the local minimum values by Eq. (2): 


$$
A S[i]=\left|\frac{H i s t[p]-H i s t[b[i]]}{p-b[i]}\right|, i=1,2, \ldots, N u m
$$

The $\mathrm{b}[\mathrm{i}]$ corresponding to the largest $\mathrm{AS}[\mathrm{i}]$ is selected as the T. Then we get a binary image containing the pupil and other parts (shown in Fig. 5(a)).

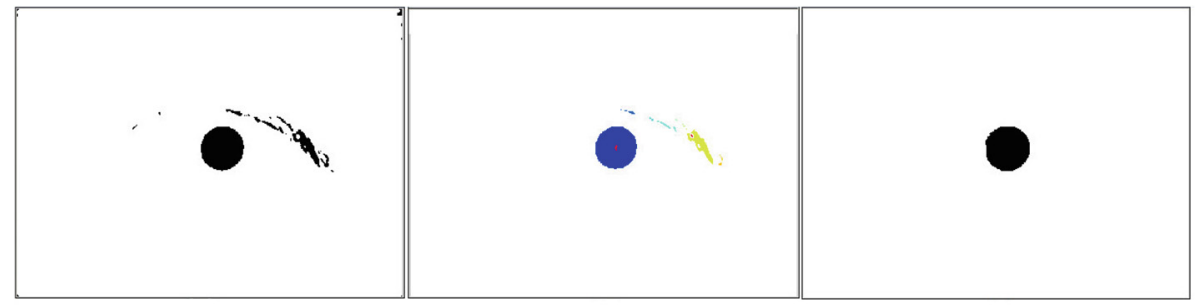

(a)

(b)

(c)

Fig. 5. Rough pupil detection: (a) Binary pupil image. (b) Connected component analysis. (c) Rough pupil.

\subsubsection{Connected Component Analysis for Rough Pupil}

It's obvious that the pupil occupies a relatively larger area and has a circular shape, which is discriminative from the noises. A connected component analysis is performed to separate and label these parts (shown in Fig. 5(b)). A Roundness Rate (RR) is a metric to measure how a area is like a circle. It's calculated as Eq. (3), in which $\mathrm{S}$ is the area and $\mathrm{C}$ the perimeter. In the circle case, the value is 1 . So the closer the $\mathrm{RR}$ is to 1 , the more likely the area is to be the pupil.

$$
R R=\frac{4 \pi * S}{C^{2}} .
$$

For every labeled part, the RR is calculated respectively. Only the most round part whose roundness rate is in the range from 0.9 to 1.1 is preserved and considered as the pupil, taking consideration that the pupil may not be a ideal circle. And then the rough pupil is located (shown as Fig. 5(c)).

\subsubsection{RANSAC to Refine Pupil Boundary}

We adopt a RANSAC-like strategy to refine the pupil boundary. Along the rough pupil boundary, we compare the gray value of every pixel on it with that of its $\mathrm{K}$ neighbor (for example $\mathrm{K}=5$ ) pixels. If the absolute difference of gray vale is less than a value to ensure continuity and consensus, then we consider that the neighbour pixel belongs to the pupil. Then we get the new and refined pupil region and calculate the geometric center $O_{p}\left(x_{p}, y_{p}\right)$ and a radius $R_{p}$ as a reference based on Eq. (4), where the $\mathrm{S}$ is the area or pixel number of the pupil.

$$
R_{p}=\sqrt{\frac{N}{\pi}} .
$$




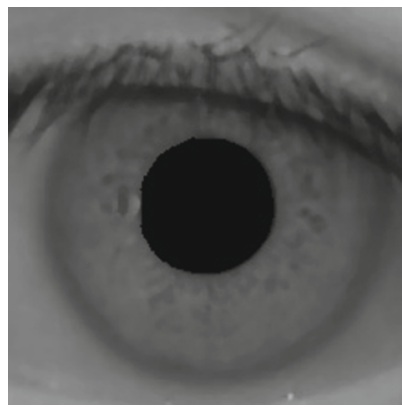

Fig. 6. ROI

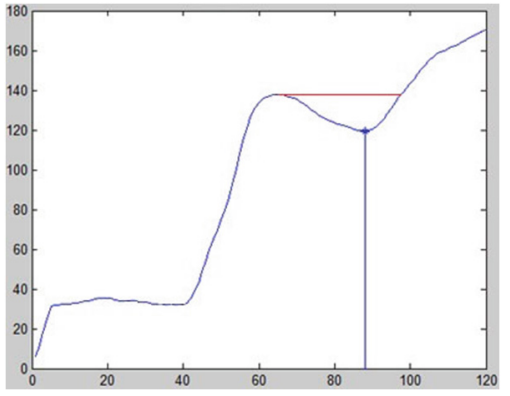

Fig. 7. Typical gray level distribution along radius direction of all effective $\theta \mathrm{s}$ : horizonal axis is the radius while vertical axis the gray value. The red line represents the width of the channel.

And by the way, we extract the region of interest (ROI,shown as Fig.6) by cropping a square with $O_{p}\left(x_{p}, y_{p}\right)$ as the center and $2 * \mathrm{~W}+1$ the edge length, where $\mathrm{W}=120$ is in the range of $\left[2 R_{p}, 3 R_{p}\right]$ from the eye image after reflections removal to cover all the iris region.

$$
R O I=I_{a}\left(x_{p}-W: x_{p}+W, y_{p}-W: y_{p}+W\right) .
$$

\subsection{Iris Limbic Boundary Detection}

\subsubsection{Iris Edge Detection}

It's usually a very difficult part to accurately locate the iris outer boundary, especially when it's weak, which means its transition zone is very wide. The famous Canny operator even fails. We refresh the definition of iris outer boundary as Fig. 7 that the gray level distribution along the radial direction from the inner boundary to the white of eye goes through three conditions: first, the transition region from pupil to iris, namely the pupil boundary or the inner boundary of iris; next, the valid iris region with rich texture information; last, the transition region from iris to the white, namely the outer boundary of iris, whose gray level distribution along the radial direction always appears as a valley and along the angle direction always a channel, which both include a series of local minima with a certain continuity and consensus.

To make it convenient to handle [18], we transform the ROI into polar coordinates as $p R O I(r, \theta)$ with the center of ROI as the pole and the left horizonal ray the pole axis (shown as Fig. 8, the radius is from $R_{p}$ to $\mathrm{W}$, whose width is $M$, while the angle is from 0 to $2 \pi$, whose length is $\mathrm{N}$ ). Given the upper and lower eyelid, we only deal with the left and right free region of ROI, namely $\theta$ is in $\left[-\frac{\pi}{6}, \frac{\pi}{6}\right] \cup\left[\frac{5 \pi}{6}, \frac{7 \pi}{6}\right]$.

Firstly, a gradient enhancer $\mathrm{P}$ is performed on each column to enhance the valley. Based on lots of experiments, a $\mathrm{P}=\left[\begin{array}{lllllll}2 & 1 & 0 & 1 & 2 & 3 & 4\end{array}\right]$ is selected. Then 


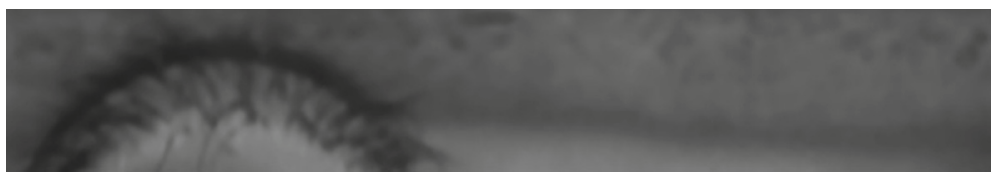

Fig. 8. pROI

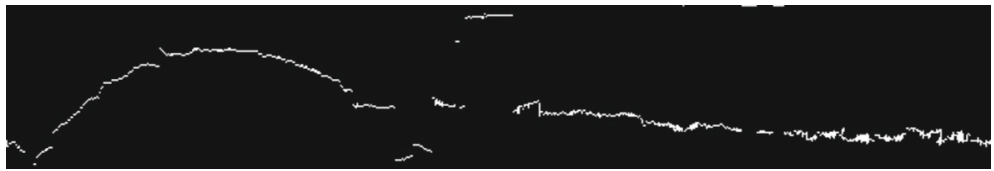

Fig. 9. Detected edge points

e extract the minimum of every column of the $p R O I(r, \theta)$ and get a Map like Fig. 9. We search the $p R O I(r, \theta)$ with the help of this Map to find those pixels of similar characteristic such as the local average gray value with these minima. Then some points far from the majority radius are rejected and only those located in a confidence interval $\left[r_{0}-\sigma, r_{0}+\sigma\right]$ (for example $\sigma=10$ ) preserved. Later, only one point is reserved in radial direction to excludes most noisy edge points [4]. And a series of valid edge points $\left\{\left(\theta_{i}, r_{\theta_{i}}\right)\right\}_{i=0}^{D}$ are got.

\subsubsection{Iris Edge Curve Fitting}

Based on the detected edge points, a circle is fitted to approximate the iris boundary [11]. This kind of method works well in the non-concentric circles cases. Firstly, we transform the points in polar coordinates to Cartesian coordinates in the original eye image in which the original point is located at the top left corner, the $\mathrm{x}$ axis is towards the right while $\mathrm{y}$ axis downward. The transformation formula is:

$$
\left\{\begin{array}{l}
x_{i}=x_{p}+\left(R_{p}+r_{\theta_{i}}\right) * \cos \left(\theta_{i}\right) \\
y_{i}=y_{p}-\left(R_{p}+r_{\theta_{i}}\right) * \sin \left(\theta_{i}\right)
\end{array}\right.
$$

Then the center $\left(x_{\text {iris }}, y_{\text {iris }}\right)$ and radius $R_{\text {iris }}$ of the fitted circle are solved based on the Least Square Error criterion [11, 14, 18,20,21].

\section{Experiment Results}

In this work, the proposed segmentation approach was tested on the internationally popular CASIA-IrisV4-Lamp, containing diverse and complex indoor NIR iris images with a high resolution of $640 \times 480$. Five hundred eye images were randomly selected and tested with two approaches $[11,19]$ and the proposed algorithm. 
Firstly, the top $60 \%$ brightest is selected as the candidate specular reflections and the real reflections in iris are picked out. The results are already shown in Fig. 3.

Next, the proposed pupil detection step is performed. Compared to traditional fixed threshold method and the thresholding method in [19], the proposed adaptive thresholding approach is more efficient, shown as Fig. 10. Then the edge of pupil is extracted as shown in Fig. 11.

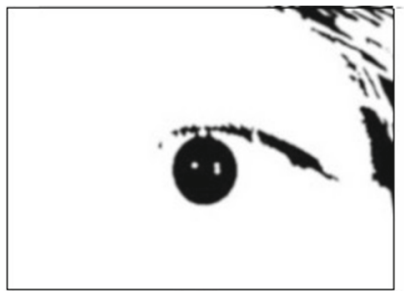

(a)

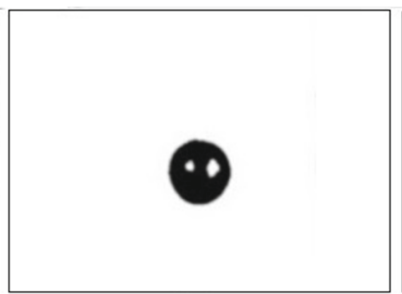

(b)

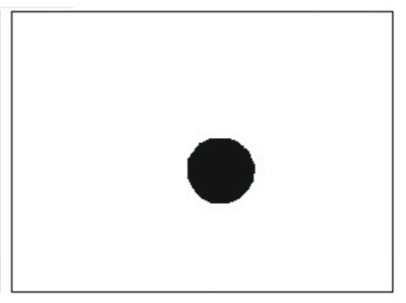

(c)

Fig. 10. Results using different thresholding methods: (a) traditional fixed threshold method. (b) threshold method in [19]. (c) Proposed threshold method.

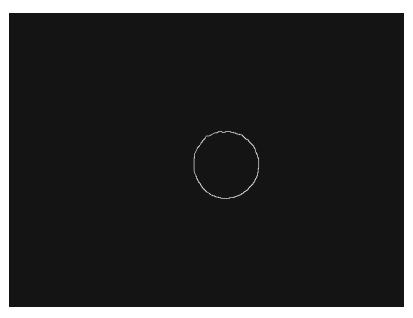

Fig. 11. Pupiledge

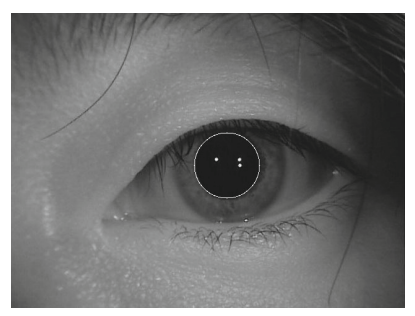

Fig. 12. Detected pupil boundary

A comparison of the three methods is shown in Fig. 13 and Table 1.

Table 1. Efficiency and accuracy analysis

\begin{tabular}{l|l|l}
\hline Method & Average running time (ms) & Accuracy $(\%)$ \\
\hline Wildes' method & 56.72 & 47.67 \\
\hline Method in [19] & 40.44 & 91.00 \\
\hline Proposed & 36.52 & 95.63 \\
\hline
\end{tabular}

In the iris outer boundary step, some results are shown in Fig. 14, and the segmentation accuracy is up to $90.52 \%$. 

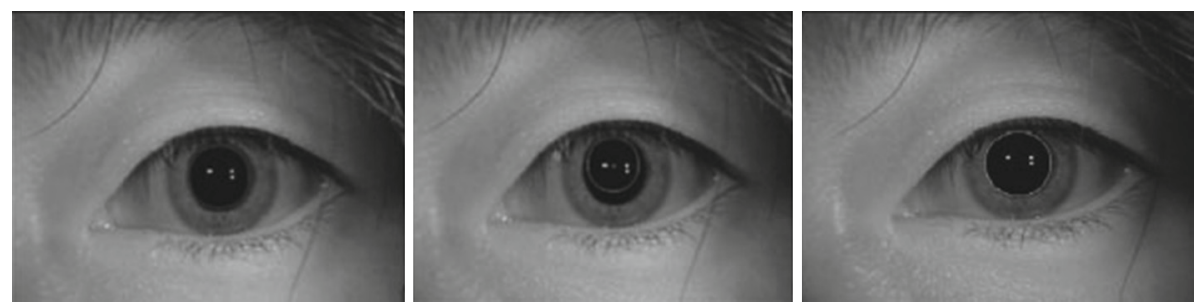

Fig. 13. Pupil localization results: (1) Left: by Wildes' method. (2) Middle: by method in [19]. (3) Right: by the proposed method.
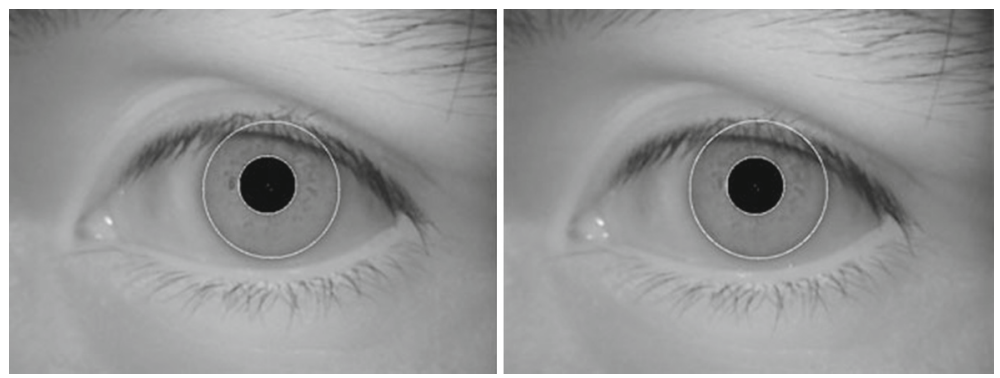

Fig. 14. Final segmentation of pupil and iris.

\section{Conclusion}

In this paper, an novel specular reflection detection method based on the neighbour gray level contrast is presented, which is in fact taking advantage of the relative distance and area between the reflection pixels and their local background. Then, a novel pupil segmentation method is proposed, which makes use of the much lower gray level than the brighter parts, like skin, white of eye and so on and also takes advantage of great difference in regularity of shape, that the pupil is much closer to a circle than other darker parts. It's just because of these characteristics of pupil that the adaptive gray-level histogram-based thresholding method can work and the pupil can be localized. It's important to detect the accurate edge points. Finally, a VLRGD method is proposed to detect the weak iris outer boundary. The proposed approach has proven quite promising in pupil and iris location according to experiment on the CASIA-IrisV4-Lamp database.

Our future work will concern more accurate and effective iris outer boundary for its great importance and eyelid detection which is currently under tested. In addition, code optimization will be done so that this algorithm can run more efficiently.

Acknowledgments. This work was supported by NSFC under grant No.61471216, Special Foundation for the Development of Strategic Emerging Industries of Shenzhen under Grant No.YJ20130402145002441, and Zhe-jiang Provincial Science\&Technology 
Research Program under Grant No. 2013C33046. Thanks for the CASIA iris database provided by Institute of Automation, Chinese Academy of Sciences, so that the present study can be made smoothly.

\section{References}

1. Daugman, J.: Biometric personal identifiation system based on iris analysis. U.S. Patent 5291560 (1994)

2. Daugman, J.: High confience visual recognition of persons by a test of statistical independence. IEEE Trans. Pattern Anal. Mach. Intell. 15(11), 1148-1161 (1993)

3. Burge, M.J., Bowyer, K. (eds.): Handbook of Iris Recognition. Advances in Computer Vision and Pattern Recognition. Springer, Heidelberg (2013). doi:10.1007/ 978-1-4471-4402-1

4. Cui, J., Wang, Y., Tan, T., Ma, L., Sun, Z.: A fast and robust iris localization method based on texture segmentation. In: SPIE Defense and Security Symposium, vol. 5404, pp. 401-408 (2004)

5. Daugman, J.: New methods in iris recognition. IEEE Trans. Syst. Man Cybern. Part B: Cybern. 37(5), 1167-1175 (2007)

6. Haindl, M., KrupiČka, M.: Unsupervised detection of non-iris occlusions. Pattern Recogn. Lett. 57, 60-65 (2015)

7. Daugman, J.: How iris recognition works. IEEE Trans. Circ. Syst. Video Technol. 14(1), 21-30 (2004)

8. Li, P., Liu, X., Xiao, L., et al.: Robust and accurate iris segmentation in very noisy iris images. Image Vis. Comput. 28(2), 246-253 (2010)

9. Shah, S., Mandowara, A., Patel, M.: Iris segmentation and recognition for human identification (2014)

10. Tan, T., He, Z., Sun, Z.: Efficient and robust segmentation of noisy iris images for non-cooperative iris recognition. Image Vis. Comput. 28(2), 223-230 (2010)

11. Wildes, R.P.: Iris recognition: an emerging biometric technology. Proc. IEEE 85(9), 1348-1363 (1997)

12. Uhl, A., Wild, P.: Weighted adaptive hough and ellipsopolar transforms for realtime iris segmentation. In: 2012 5th IAPR International Conference on Biometrics (ICB), pp. 283-290. IEEE (2012)

13. Popplewell, K., Roy, K., Ahmad, F., et al.: Multispectral iris recognition utilizing hough transform and modified LBP. In: 2014 IEEE International Conference on Systems, Man and Cybernetics (SMC), pp. 1396-1399. IEEE (2014)

14. Leo, M., Marco, T.D., Distante, C.: Highly usable and accurate iris segmentation. In: 2014 22nd International Conference on Pattern Recognition (ICPR), pp. 24892494. IEEE (2014)

15. Kaur, N., Juneja, M.: Segmentation approach for iris recognition in less constrained environment. In: Maharatna, K., Dalapati, G.K., Banerjee, P.K., Mallick, A.K., Mukherjee, M. (eds.) Computational Advancement in Communication Circuits and Systems. Lecture Notes in Electrical Engineering, vol. 335, pp. 481-490. Springer, India (2015)

16. Sahmoud, S.A., Abuhaiba, I.S.: Efficient iris segmentation method in unconstrained environments. Pattern Recogn. 46(12), 3174-3185 (2013)

17. Daugman, J.: New methods in iris recognition. IEEE Trans. Syst. Man Cybern. Part B: Cybern. 37(5), 1167-1175 (2007)

18. He, Z., Tan, T., Sun, Z., et al.: Toward accurate and fast iris segmentation for iris biometrics. IEEE Trans. Pattern Anal. Mach. Intell. 31(9), 1670-1684 (2009) 
19. Lin, Y., Qu, Z., Zhang, Y., et al.: A fast and accurate pupil localization method using gray gradient differential and curve fitting. In: Proceedings of the 4th International Conference on Computer Engineering and Networks, pp. 495-503. Springer, Heidelberg (2015)

20. Tan, C.W., Kumar, A.: Automated segmentation of iris images using visible wavelength face images. In: 2011 IEEE Computer Society Conference on Computer Vision and Pattern Recognition Workshops (CVPRW), pp. 9-14. IEEE (2011)

21. Proenca, H.: Iris recognition: on the segmentation of degraded images acquired in the visible wavelength. IEEE Trans. Pattern Anal. Mach. Intell. 32(8), 1502-1516 (2010)

22. Cui, J., Wang, Y., Tan, T., et al.: A fast and robust iris localization method based on texture segmentation. In: International Society for Optics and Photonics, Defense and Security, pp. 401-408 (2004)

23. CASIA iris image database. http://www.cbsr.ia.ac.cn/Databases.htm 